\title{
Man is What He Eats: A Research on Hinglish Sentiments of YouTube Cookery Channels Using Deep learning
}

\author{
Suraj Kumar Donthula, AbhishekKaushik
}

\begin{abstract}
Our study focuses on the sentiment analysis of Hinglish comments by multi-label text classification on cookery channels of YouTube using Deep learning. Multi-layer perceptron $(M L P)$ with different parameters was implemented in our study to investigate the various sentiments in the comments. We have modelled and evaluated MLP by varying the number of neurons, layers, optimizers, activation functions with the various feature engineering methods such as $t$-idf, count vectorizer, pre-trained embeddings and customizedembeddings. These experiments were conducted on two datasets they are Kabita's Kitchen and NishaMadhulika's dataset. From the investigation, we concludedKabita's Kitchen dataset has the highest accuracy 98.53\% and NishaMadulika'shas $98.48 \%$ accuracy in MLP.This outcome of the experiment was evaluated based on careful analysis on tests conducted during our study.
\end{abstract}

Keywords: Cookery channels,Multi-layer perceptron, Hinglish, Sentiment Analysis.

\section{INTRODUCTION}

Food and family are possibly India's two biggest obsessions. In this fast-growing world, many people are leaving their family and moving to cities for jobs, studies and businesses which enable peoples to learn cooking. Though there are many mediums to learn cooking but due to the available access of internet in India lots of people are opting for cookery channels in YouTube. This led to sky rocketed increase in number of cookery channels based on Indian food. Therefore, there is increase in competition for youtubers to satisfy the viewers by creating good quality content. To grow as a successful channel, interaction between the users and creators is more important and specially for this cookery channels because the result of following cooking from a cookery channel is instant, this leads to a lot of doubts while in process. These comments are one the social metrics that will help your video to be ranked higher in the search engine result if you get more of it. It's one of the factors that affects it other than watch time, views, likes and many others.

Comments are very important as it gives the content creator a way to communicate with its viewers and subscribers. It's very important to maintain the relationship between content creator and its viewers. The social interaction that mainly being used is the comment section. It's also provided a way for the content creators to get fresh

Revised Version Manuscript Received on 16 September, 2019.

Suraj Kumar Donthula,School of Computing, Dublin Business School, D02 WC04 Dublin, Ireland.

(Email: Suraj.don46@gmail.com)

AbhishekKaushik, ADAPT Centre, School of Computing, Dublin City University, D09 W6Y4 Dublin, Ireland.

(Email: abhishek.kaushik2@mail.dcu.ie) new ideas of the future content. It will help you to improve your social skill and give the opportunity to appreciate and reward you viewers for their supports to your videos and channel. This persuaded us to perform research on Indian cookery channels and discover approaches to help the youtubers and suggest them solutions to for interactivity [1]. During our literature review we found a study has been conducted on the cookery channel by creating and training dataset using machine learning models to comprehend and examine the user's necessities from thevideo. We took that study of Gagandeep et al. as a baseline model and applied deep learning MLP models with different parameters on the similar dataset to improve accuracy [1].

Sentiment Analysis is a field of Natural Language Processing (NLP) that develops a framework which is used to recognize and extricate judgement inside content [2]. With the assistance of this frameworks, this unstructured data could be naturally changed into organized information of general sentiments about items, administrations, brands, legislative issues, or any theme that individuals can express feelings about. These writings are normally troublesome, tedious and costly to investigate, comprehend, and sort through. Aside from the feelings, for sentiment analysis language additionally assumes a significant job, as a rule remarks by individuals are in blend of different dialects. This Multilingual feeling examination can be a troublesome assignment. Ordinarily, a great deal of pre-handling of data is required which is one of the difficulties. Comments under cookery videos are generally in Hinglish which means Hindi language written in English content with numerous words from English jargon. Since Hinglish essentially addresses mapping the information from English to any morphological language doing sentiment analysis is an unprecedented test. The absence of assets for the Hinglish language brings difficulties going from storing up to time of datasets. We reacted to this test and dealt with the Hinglish language.

This study is partitioned into different sections such as literature review, methodology, results, discussions and conclusion. Literature review includes the previous work done on the sentiment analysis, text classification using machine learning and deep learning. Methodology incorporates the information about the process followed, type of feature engineering and about the model and its parameters used to increase the accuracy. In results, we provided the 
results obtained by the model with various combinations of parameters with different types of vectorizers are presented. In discussion, we discussed about the outcome has been outlined in discussion section. In conclusion we concluded the work which we have done.

\section{LITERATURE REVIEW}

This section is a review of the studies related to the sentiment analysis and text classification approaches, therefore, in this we discuss and compare some of the research topics, applied techniques, feature selection methods, limitations, computational difficulties and model performances.

\section{A. Deep learning and decision trees approach used for} Sentiment analysis

This paper exhibits the new approaches of deep learning and decision trees to deal with the movie reviews for Sentiment analysis [3]. The data set used consists of 50,000 IMDB movie reviews, specially selected for sentiment analysis. random forest and deep learning method such as deep recurrent neural networks are used for the message classification. The bag of words model with random forest gives an accuracy of $85.2 \%$ and deep learning (i.e., recurring neural networks) gives an accuracy of $90.3 \%$. The given work shows that surface analysis of a text is not enough for sentiment analysis. Of course, simple methods can be used for systems where high speed and resources are important. But for results-oriented services it would be not enough. Information [3].

\section{B. Detecting inappropriate comments by Text Classification.}

In this paper, process involves performing text analysis when a user posts a comment on certain article and judge whether a comment is good or bad[4]. They used natural language processing to identity inappropriate content and compared a group of algorithms and assess distinctive capabilities to show signs of improvement precision for characterization. Swedish language data set manually labelled by two students at LTH and English language data set from a Kaggle competition detecting insults in social commentary are used for this process [4]. By using different feature selection and applying the machine learning algorithms by different combination they got results and thus found that SVM and logistic regression are performing similar, however logistic regression scored slightly higher and has the advantage of providing a probability score describing the certainty that a

comment belongs to one of the two classes.

\section{Classification of Political logs Sentiment by Mining}

This project deals with the web-logs data and discusses the prevailing techniques and their usage for sentiment classification. The data is extracted from the website1, which is political web log. Naive Bayes and Support Vector Machines are the algorithms used for this classification. They conducted the experiment by creating 5 collections, four are classified by Naive Bayes and the remained by Support vector machines. Each collection is used to evaluate the effectiveness of known aspect of the sentiment classification
[5]. On an average we get accuracy for Naive Bayes as $78.06 \%$ and for Support Vector Machines it is $75.47 \%$.

D. Automatic web page categorization using text classification methods

The goal of this thesis is to use Natural language processing and Machine learning tools for the text classification to implement the web-page categorization [6]. This process involves extracting textual, natural language content from web page, encoding the document as a feature vector with natural language processing methods. Data from 'Whaam' on which user stores links of different categories, has used for this project. Data in a webpage is divided accordingly as per development of webpage and classifications applied on every division. Support Vector Machines (SVM), k-nearest neighbor $(\mathrm{kNN})$, Multinomial Naive Bayes (MNB), Term-Weighted Complementary Naive Bayes (TWNCB) and the Cavnar-Trenkle N-Gram-based Classifier (N-Gram) are the algorithms used for implementing the classification. The results show that they are not terrible, suggesting that with the honing of these methods leads to increase in the success rate [6].

\section{E. Classification of comments with Deep neural network based on attention mechanism}

This paper proposes a CNN-Attention network based on Convolutional Neural Network with Attention (CNNA) mechanism in which all, information between words for context can be expressed by using different sizes of convolution kernels after this, an attention layer is added to convolution network to obtain semantic codes which has the attention probability distribution of input text sequences [7]. Furthermore, weights of text representing information are calculated and finally, the SoftMax is used to classify emotional sentences. It is proposed because it is very difficult to represent the text information with shallow network, and it is time-consuming for using deep neural network[7]. In this method the features of different context information can be extracted thereby reducing the depth of the network and the accuracy is improved. The dataset used is the COAE2014 ${ }^{1}$ task 4 weibo data set. The outcome of this study tells that the Text semantic information and text features are extracted using bottom layer and experimental results should that the performance of the model is $95.15 \%$.

\section{F. An Experimental Comparison of Text Classification} Techniques

A lot of important status is given to archives management as there is skyrocketing of documents in computerized structure and the resulting need to get to them in adaptable ways. So, text classification field became the one of the more centered arenas in AI research. One of the key procedures used to deal with and sort out content information is text classification which is the undertaking of marking common language writings with topical classifications from a predefined set [8]. The main aim of the thesis is to compare the performance of current text classification techniques, from standard SVM-based, statistical and multilayer 
perceptron (MLP) models to the deep learning models such as convolutional neural networks and their combination with graph theory[8]. Rotten Tomatoes, 20 Newsgroups and Reuters data of Volume 1 are the datasets used for this comparison. They concluded two results that convolutional neural networks with graph theory perform with greater test accuracy when compared to classical CNNs, SVM-based models and statistical baseline models. Second, and more surprisingly, simpler MLP models still outperform recent deep learning techniques despite having fewer parameters.

G. Spam detection in online social networks by Deep learning

The aim of this thesis is to detect the spam in the social networking sites by using deep learning approaches. In this Word2 Vec is used for feature selection and then deep learning methods are used to distinguish the spam and the no spam tweets [9]. For the dataset, they collected 5-days tweet data from Twitter's Streaming API. The dataset contains about 10 million tweets. These tweets are processed by JSON designing and every line in this work is handled as a target. Different measures such as Precision, Recall, and F-measure are used to evaluate the performance of the methods[9]. Multilayer Perceptron, Random Forest J48 and are the algorithms used for the classification. The results show that the precision value of Multilayer perceptron MLP is $92 \%$. The worst result of this measure is obtained by Naive Bayes Classifier. The process on these tweets exhibit that of this deep learning approaches outperform the classical approaches.

H. Sentiment Classification: Feature Selection Based Approaches Versus Deep Learning

This paper compared the performance of feature selection-based approaches and deep learning architecture, convolutional neural network, long short-term memory network, and long-term recurrent convolutional network. For text classification, traditional method for feature selection such as bag-of-words is used, these features are given as input to the model such as Support Vector Machines (SVM) and neural networks. The hindrance to the bag-of-words feature selection method is enormous loss of data during preparing stage since the document depends on the recurrence of a word which doesn't consider the significance and order of the words with low frequency, so to conquer this issue a new technique called word embedding has been developed for text mining [10]. In this paper experiments are conducted on 4 datasets which are having different characteristics they are IMDB movie review dataset, subset of Sentiment $140^{2}$ dataset, Nine Public Sentiment dataset consisting of positive and negative reviews from such domains as laptop, music etc. and Multi-domain dataset which contains reviews collected for many different products of Amazon.com reviews. For analyzing deep learning techniques used are convolutional neural network, long short-term memory network, and long-term recurrent convolutional network and for feature selection approach, a hybrid method which combined bag-of-words features and averaged word embedding features are used. At first, they classified using SVM Classifier for both feature selection models and then deep learning models and hybrid deep learning models are used. The results show that at least one deep learning model outperform all feature selection-based methods. The hybrid model here it is CNN+LSTM is the best performer among all for IMDB dataset, CNN performs best than the other methods for nine public sentiment and multi-domain dataset. The only dataset where IG and Word Embed-dings given better results is on Sentiment140 dataset. They concluded that we can achieve more better results in sentiment classification by using deep learning models with fine-tuned semantic word embed-dings [10].

\section{Research on hot news classification algorithm based on} deep learning

Now-a-days people are more interested towards the hot problem in news because of the advancements in the arena of network applications. So, there is need to classify the hot problem in the news. This proposal aims to study the various deep learning approaches in text categorization, combined with the characteristics of the news text, put forward the double Bi-Gated Recurrent Unit (GRU) + attention deep learning model to predict the hotspots. Since the methods such as Many methods based on machine learning, such as Naive Bayes, Support Vector Machine (SVM), classification tree and vector-based features, cannot study the semantics the accuracy of the classification is not high. Due to this kind of issues and increase in the sub-fields the classification has become more challenging. This paper proposes a new hybrid deep learning model, which can be used for text categorization, namely BiGRU + attention mechanism. This method has higher precision when compared with the classical approaches of text classification because of considering the full context of data and focus problems. The data is fetched from the sohu website crawl, dated from October 20, 2015 to April 25, 2016, includes financial news, social news and other hot news from sohu buzz [11]. The outcome show that the predicted results of our model are superior to the traditional model of deep learning. This method has higher precision when compared with the classical approaches of text classification because of considering the full context of data and focus problems [11].

As per the literature review studied, researchers worked on detecting positive and negative comments and mostly binary text classification whereas our study is related to sentiment analysis by implementing multi label text classification. We also found that more research has been done on a single language, and very few works has been done on a mix of two languages. While conducting a research we discovered a similar research has been already done by Gagandeep et al. on cookery channels of YouTube and achieved highest accuracy of $76.56 \%$. In this research of Gagandeep et al., clustered a dataset by using API and sentimental analysis has been performed by using machine learning algorithms. Now we considered Gagandeep et al. models as baseline models and implemented neural network-based model multi-layer perceptron with different parameters to increase the performance. 


\section{METHODOLOGY}

In this section, the process followed for sentiment analysis by multi-label classification is discussed.

\section{a. Dataset Preparation}

As our aim is to increase the performance of baseline models which is achieved in Gagandeep et al. paper, we also used the same datasets. The data set [12] is downloaded link ${ }^{3}$ which is created by Abhishek et al.

The dataset contains the pre-processed (removal of stop words, empty values, numbers, special characters and punctuation, converting into lower case, tokenization and stemming [1]) Hinglish comments of top cookery channels, named NishaMadhulika Cooking Channel and Kabita's Kitchen.

The dataset was divided into seven categories with an equivalent number of trails, 700, each. The total data in all the datasets is 9800[1].

Downloaded glove.twitter.27B.200d embedding and used for feature engineering.

According to the Gagandeep et al. paper there are seven categories of comments such as

Label-1 Gratitude

Label-2 About recipe

Label-3 About video

Label-4 Praising

Label-5 Hybrid

Label-6 Undefined

Label-7 Suggestion or queries

The initial step in this is selection of data, loading a dataset and performing basic pre-processing steps. The dataset is then splitted into train and test sets.

Downloaded glove.twitter.27B.200d embedding data also and used as part of feature engineering.

\section{b. Feature Engineering}

It is the most significant step in the text classification. In this the dataset is converted into flat features i.e., by converting the data into a matrix used to train a model. This progression likewise raw incorporates the way toward making new highlights from the current information.

In this, information will be changed into feature vectors and new features will be made utilizing the current dataset. We will execute the accompanying various thoughts to acquire important features from our dataset.

Count Vectorization: It is a matrix notation of data in which there are different types of representations, in this row represents a document, column represents a term and cell represents a count of frequency in a document [13].

TF-IDF Vectorization: It represents the general significance of a term in the archive and the whole corpus. The features weighting mechanism commonly used are as Term frequency and inverse document frequency (TFIDF). Kaushik et $a l$. described in detail about the features weighting mechanism techniques and consequence in machine learning model [13].

TF-IDF score is made by two terms: the first figures the standardized Term Frequency (TF), the subsequent term is the Inverse Document Frequency (IDF), registered as the logarithm of the quantity of the reports in the corpus divided by the quantity of documents where the particular term shows up.

Word Embedding: A word embedding is a form of representing words utilizing a thick vector portrayal. The situation of a word inside the vector space is found out from content and depends on the words that encompass the word when it is utilized. Word embeddings can be prepared utilizing the information corpus itself or can be created utilizing pre-prepared word embeddings, for example, Glove, FastText, and Word2Vec.

\section{c. Model Training}

The last step in the classification system is to prepare a classifier utilizing the features discovered in the previous step. There are a wide range of decisions of machine learning models which can be utilized to prepare a final classifier. Our aim to compare the performance of multi-layer perceptron in multi label text classification with the traditional baseline machine learning models [10].

Multi-Layer Perceptron: MLP is a feedforward artificial neural network that generates a set of outputs from a set of inputs. An MLP is characterized by several layers of input nodes connected as a directed graph between the input and output layers. It is a field that explores how basic models of brains can be utilized to illuminate troublesome computational assignments like the predictive modelling as we find in machine learning.

Networks of Neurons: A layer has multiple neurons and a network has multiple layers. These neurons are masterminded into system of neurons. The arrangement of neurons in a network is called the network topology.

Input Layers: The base layer that takes input from your dataset is known as input layer, since it is the uncovered piece of the system it is also called as visible layer. Regularly a neural system is drawn with a visible layer with one neuron for every information in your dataset. These are not neurons as depicted above, however basically pass the info esteem through to the following layer.

Hidden Layers: Since these are not directly exposed to the input these are called Hidden layers. The basic architecture of neural network to have a single neuron in the hidden layer that directly outputs the value.

Output Layer: The layer which is known for providing a output by a vector that required for the issues is the final layer called as output layer.

Activation Function: The weighted sources of info are summed and went through an actuation work. It is a basic mapping of summed weighted contribution to the output of the neuron.

When output is 0 and 1 we use a non-linear function like logistic also called the sigmoid function, when the output is in range of -1 and +1 we use tangent function called tanh.

More as of late the rectifier activation function has been appeared to give better outcomes.

Loss Functions: Loss function is a significant part in neural systems, which is utilized to gauge the irregularity between predicted and actual labels. It is a non-negative

Published By: 
value, where the power of model increments alongside the diminishing of the estimation of this function.

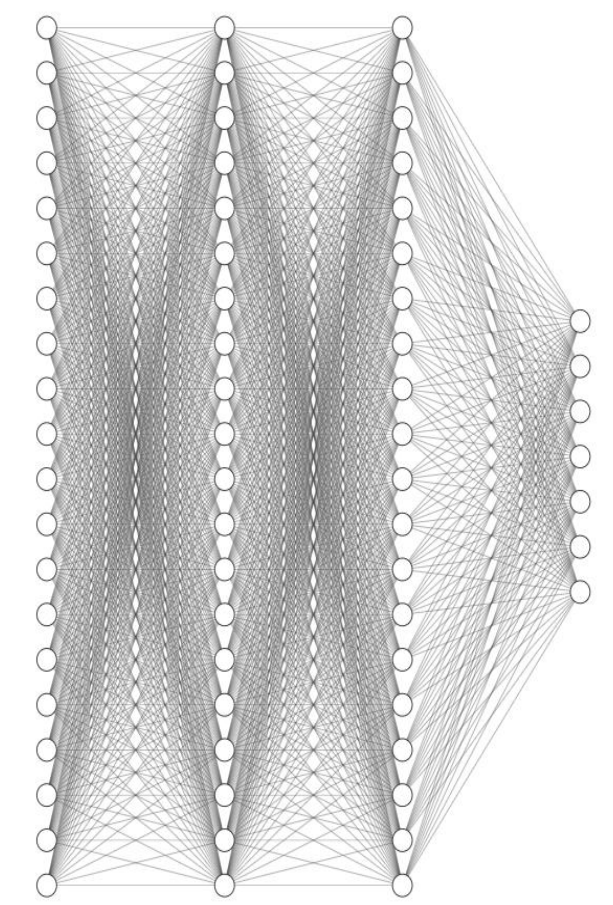
Figure 1- Multi layer Neural Network with 4 layers 20
neurons per layer and 7 neurons for output layer

\section{RESULTS}

When a neural system has been prepared it very well it can be utilized for the classification. You can group the test data to assess the performance of the model on concealed information. The system topology and the last step of weights is all that you must spare from the model.

The outcome of this experiment was obtained by Multi-layer perceptron over both the datasets using different feature vectorizers like count, tf-idfvectorizer, pre-trained embeddings and custom embeddings ${ }^{4}$. The Experiment is conducted on Multi-layer perceptron with different combinations of parameters such as layers, number of neurons, activation, optimizer functions. The metrics used to is accuracy.

On Kabita's dataset the outcome we get that Multi-layer perceptron with count vectorizer with optimizer and activation function as 'adam' and 'tanh' has the highest accuracy $98.53 \%$ (Table-1). It is very close to TF-IDF vectorizer with optimizer and activation function as 'adam' and 'leaky relu' with $98.13 \%$. Count vectorizer has an average of $>90 \%$ with all the combinations of parameters. Optimizer 'adam' gives an average accuracy of $>90 \%$ whereas 'sga' optimizer gives accuracy of $<60 \%$. Comparatively Embeddings also gives less accuracy than tf-idf and count vectorizer.
Table 1: Results on Kabita's Kitchen dataset.

\begin{tabular}{|c|c|c|c|c|c|}
\hline Model & $\begin{array}{r}\text { layer } \\
\mathrm{s} \\
\end{array}$ & $\begin{array}{r}\text { neuron } \\
\mathrm{s} \\
\end{array}$ & $\begin{array}{l}\text { Optimize } \\
\mathrm{r}\end{array}$ & $\begin{array}{l}\text { Activatio } \\
\mathrm{n}\end{array}$ & $\begin{array}{r}\text { Accurac } \\
\mathrm{y} \\
\end{array}$ \\
\hline \multirow{5}{*}{$\begin{array}{c}\text { count } \\
\text { vectorizer }\end{array}$} & 2 & 20 & adam & $\tanh$ & 98.39 \\
\hline & 3 & 20 & adam & $\tanh$ & 98.39 \\
\hline & 3 & 20 & adam & leaky relu & 98.48 \\
\hline & 3 & 15 & adam & $\tanh$ & 98.53 \\
\hline & 3 & 20 & adam & $\tanh$ & $\mathbf{9 8 . 5 3}$ \\
\hline \multirow{5}{*}{ Tf-idf } & 2 & 20 & adam & $\tanh$ & 97.78 \\
\hline & 3 & 20 & adam & $\tanh$ & 97.81 \\
\hline & 3 & 15 & adam & $\tanh$ & 97.84 \\
\hline & 3 & 20 & adam & relu & 97.99 \\
\hline & 3 & 20 & adam & leaky relu & 98.13 \\
\hline \multirow{5}{*}{$\begin{array}{l}\text { Pre-trained } \\
\text { embedding } \\
\mathrm{s}\end{array}$} & 2 & 20 & adam & $\tanh$ & 77.2 \\
\hline & 2 & 20 & adam & leaky relu & 77.25 \\
\hline & 3 & 20 & adam & leaky relu & 78.16 \\
\hline & 2 & 20 & adam & relu & 78.27 \\
\hline & 3 & 20 & adam & relu & 78.54 \\
\hline \multirow{5}{*}{$\begin{array}{c}\text { custom } \\
\text { embedding }\end{array}$} & 2 & 20 & adam & leaky relu & 88.24 \\
\hline & 3 & 20 & adam & leaky relu & 89.48 \\
\hline & 2 & 20 & adam & $\tanh$ & 89.69 \\
\hline & 2 & 20 & adam & relu & 89.69 \\
\hline & 3 & 20 & adam & $\tanh$ & 89.91 \\
\hline
\end{tabular}

On Nisha's dataset we found the similar results, multi-layer perceptron with count vectorizer with optimizer and activation function as 'adam' and 'tanh' has highest accuracy $98.48 \%$ (Table-2). For the same parameters with Tf-idf we got an accuracy of $98.22 \%$. On this dataset both tf-idf and count vectorizers show an average accuracy of $>90 \%$. Similar results are also observed in case of embeddings i.e., less than tf-idf and count vectorizer.

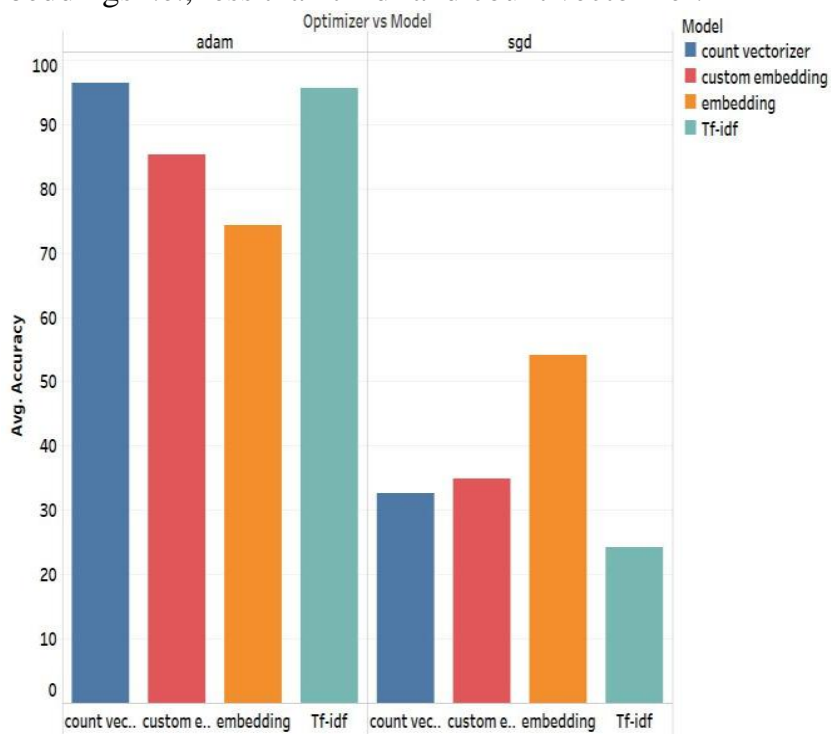

Figure 2- Bar graph showing accuracies on Kabita's dataset using tf-idf, count frequency vectorizer, pre-trained embedding and own embedding with their optimizers. 


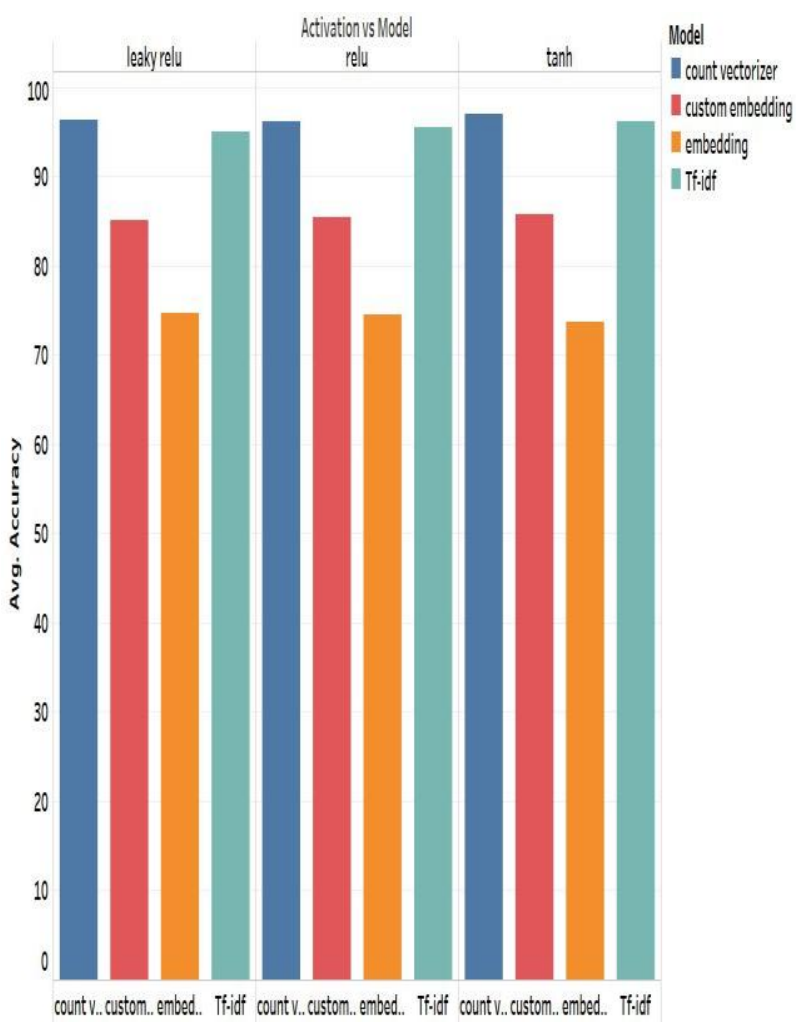

Figure 3- Bar graph showing accuracies on Kabita's dataset using tf-idf, count frequency vectorizer, pre-trained embedding and own embedding with their activation function.

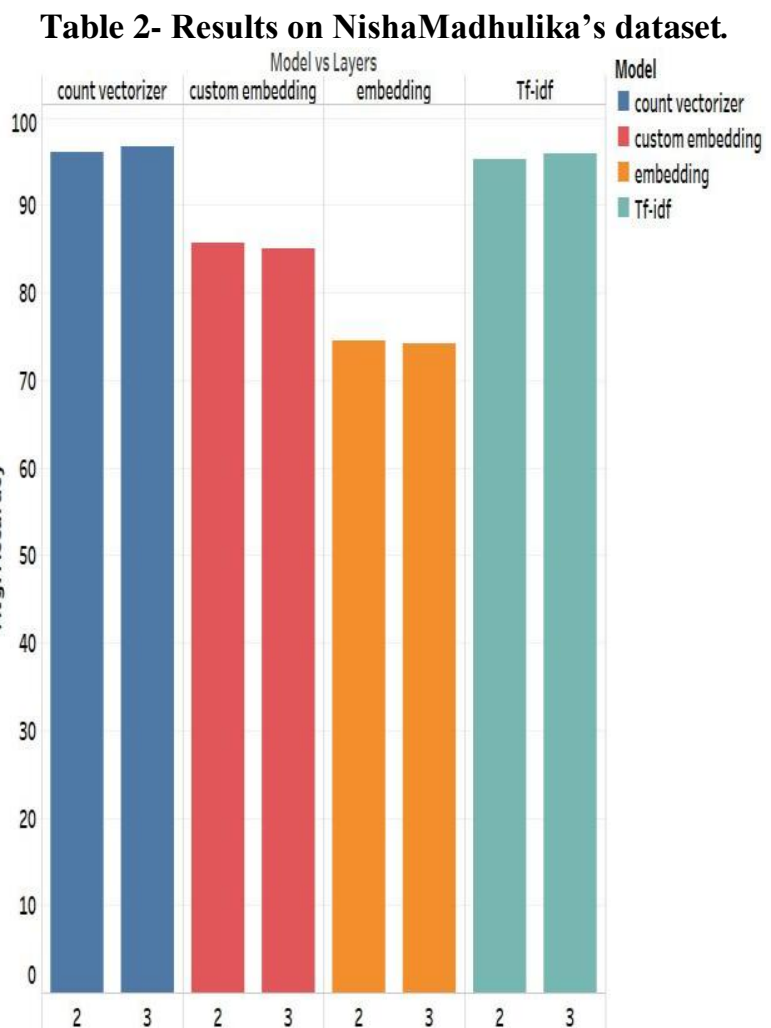

Figure 4-Bar graph showing accuracies on Kabita's dataset using tf-idf, count frequency vectorizer, pre-trained embedding and own embedding with their Number of layers.

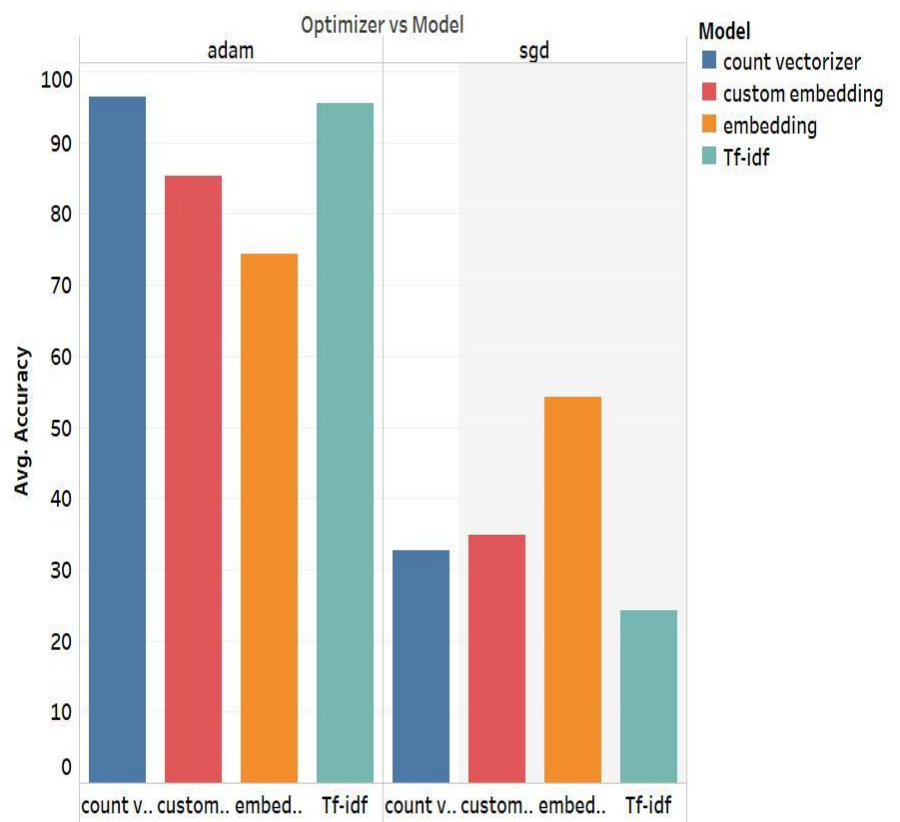

Figure 5- Bar graph showing accuracies on NishaMadhulika's dataset using tf-idf, count frequency vectorizer, pre-trained embedding and own embedding with their optimizers.

Table 3: Results on NishaMadhulika's Kitchen dataset.

\begin{tabular}{|c|c|c|c|c|c|}
\hline Model & layers & neurons & Optimizer & Activation & Accuracy \\
\hline \multirow{5}{*}{$\begin{array}{c}\text { count } \\
\text { vectorizer }\end{array}$} & 2 & 20 & adam & leaky relu & 98.23 \\
\hline & 3 & 15 & adam & $\tanh$ & 98.29 \\
\hline & 3 & 20 & adam & relu & 98.39 \\
\hline & 3 & 20 & adam & $\tanh$ & 98.42 \\
\hline & 3 & 20 & adam & $\tanh$ & 98.48 \\
\hline \multirow{5}{*}{ Tf-idf } & 3 & 20 & adam & relu & 97.93 \\
\hline & 2 & 20 & adam & $\tanh$ & 98.02 \\
\hline & 3 & 20 & adam & $\tanh$ & 98.1 \\
\hline & 3 & 20 & adam & leaky relu & 98.1 \\
\hline & 3 & 20 & adam & $\tanh$ & 98.22 \\
\hline \multirow{5}{*}{$\begin{array}{l}\text { Pre-trained } \\
\text { embedding }\end{array}$} & 3 & 20 & adam & relu & 77.17 \\
\hline & 3 & 20 & adam & leaky relu & 77.34 \\
\hline & 2 & 20 & adam & $\tanh$ & 77.63 \\
\hline & 2 & 20 & adam & leaky relu & 77.93 \\
\hline & 2 & 20 & adam & relu & 78.77 \\
\hline \multirow{5}{*}{$\begin{array}{l}\text { custom } \\
\text { embeddings }\end{array}$} & 2 & 20 & adam & $\tanh$ & 89.73 \\
\hline & 2 & 20 & adam & leaky relu & 90.34 \\
\hline & 3 & 20 & adam & relu & 91.2 \\
\hline & 2 & 20 & adam & relu & 91.38 \\
\hline & 3 & 20 & adam & $\tanh$ & 91.83 \\
\hline
\end{tabular}




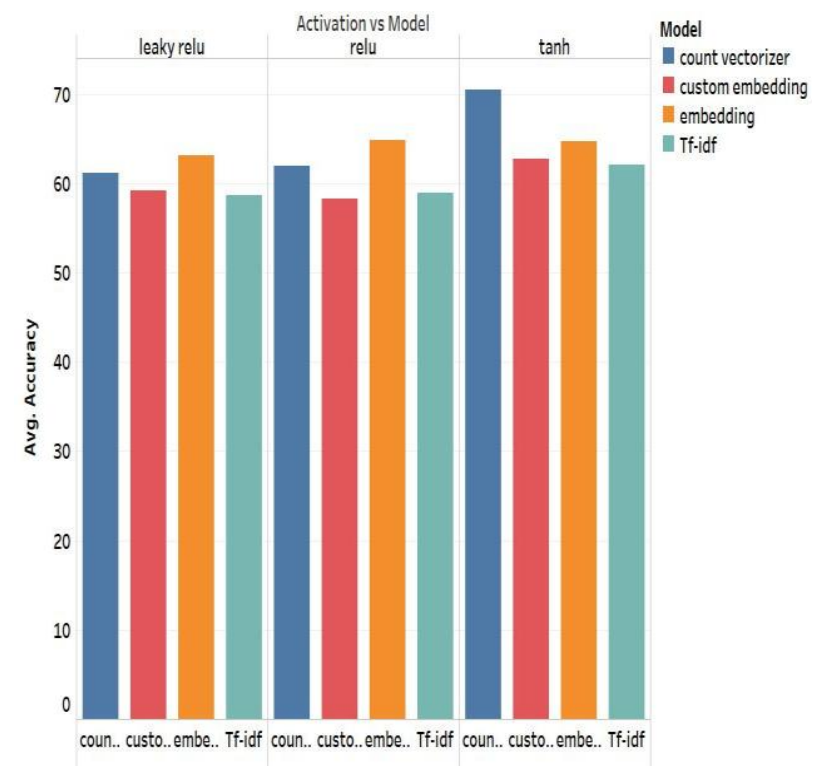

Figure 6-Bar graph showing accuracies on NishaMadhulika's dataset using tf-idf, count frequency vectorizer, pre-trained embedding and own embedding with their activation functions.

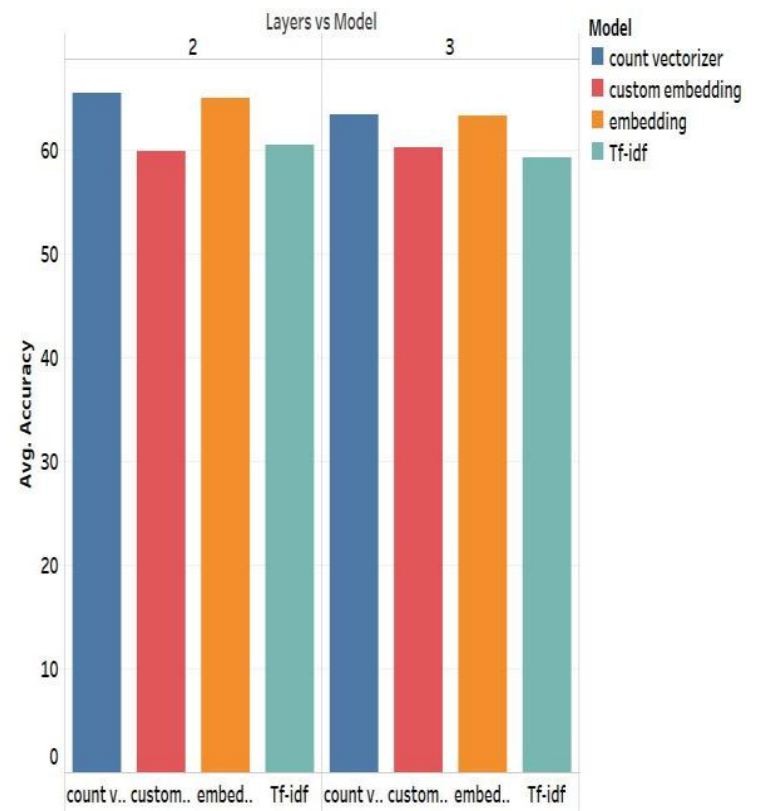

Figure 7-Bar graph showing accuracies on NishaMadhulika's dataset using tf-idf, count frequency vectorizer, pre-trained embedding and own embedding with their number of layers.

\section{DISCUSSION}

The objective was to locate the best MLP which gives higher performance than the baseline models and, in view of our examination, it was discovered that the Multi-Layer perceptron model performed better on both the datasets. Numerous examinations have been finished on machine learning and deep learning models on sentiment analysis and content classification but in this we used multi-layer perceptron with different combinations of parameters and made comparisons on them. The purpose for utilizing these models was to check how a neural network model works on Hinglish comments multi-label text classification.

From the results the outcome we get that tf-idf and count vectorizer gives a highest accuracy. We also found less performance in case of word embeddings because our research is based on Hinglish comments and we use English pre-trained embeddings and embedding from our corpus also gives low accuracy because of the dataset size.

As per experiment conducted on different parameters, we have visualized the results, we have made visualizations with average accuracy and parameters used on both datasets. From the visualization (Figure-2 \& Figure-5) we can say that 'adam' optimizer gives better results on both the datasets. From (Figure-3 \& Figure-6) we can also conclude that the performance of activation function depends upon the feature engineering techniques because the (Figure-2 \& Figure-5) shows different accuracies for all the activation functions. We also observed that increase in the layers (Figure-4 \& Figure-7) and epochs shows better results, but with the increase in the size of dataset the time consumed for training will be high, time constraint should also be taken care when choosing the number of layers.

To increase the number of subscribers, one must be in touch with the followers, our model enables the youtuber to predict the label which can help to understand the user's requirement. In order to improve our exploration and give the different cookery channels on YouTube to construct their supporters base, we are intended to develop model with the Rest API to make the process automated. Building the Rest API is the future enhancement of our present investigation.

\section{CONCLUSION AND FUTURE WORK}

Commitment and engaging with users is an immense trendy expression in the internet based life space. Social media brands are regularly entrusted with showing their commitment with their users on every social network they are dynamic on, yet frequently comments on a YouTube channel are left unanswered. It's similarly as essential to react to the comments of YouTube on your channel for what it's worth to react to a wall post on your Facebook Page. Here we chose cookery channels based on Indian cuisines as most of the Indians are living abroad and migrating to nearest cities. Therefore, our research is on doing sentiment analysis on the YouTube cookery channels. Since we found already a study has been done on this topic, in that they used machine learning algorithms we used these models as baseline and applied multi-layer perceptron model with different combinations of parameters. We got an accuracy of $98.53 \%$ on Kabita's dataset and $98.48 \%$ on NishaMadhulika's dataset by multi-layer perceptron with the count vectorizer. By this it can be concluded that multi-layer perceptron with count vectorizer provides better results than the baseline models.

For future, we are planning to create a pre-trained Hinglish embedding and increase the size of the dataset by adding recent comments by doing this we can get better results for models with embeddings. We additionally intending to apply advanced deep learning models and perform correlations by which we discover better model to develop Rest API, which would push Youtubers to consequently isolate the users'

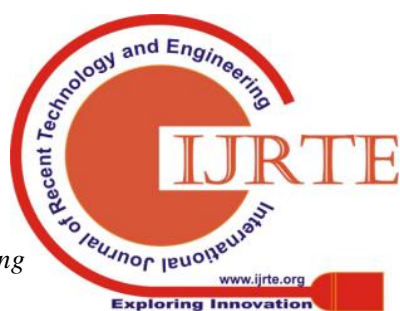


remarks and help them to comprehend the necessities of the users [10].

\section{REFERENCES}

1. G. Kaur, A. Kaushik, and S. Sharma, "Cooking Is Creating Emotion: A Study on Hinglish Sentiments of Youtube Cookery Channels Using Semi-Supervised Approach," Big Data and Cognitive Computing, vol. 3, no. 3, p. 37, Jul. 2019.

2. A. Kaushik and S. Naithani, "A Comprehensive Study of Text Mining Approach," International Journal of Computer Science and Network Security (IJCSNS), vol. 16, no. 2, p. 69, 2016.

3. A. S. Zharmagambetov and A. A. Pak, "Sentiment analysis of a document using deep learning approach and decision trees," in Proceedings of the 2015 12th International Conference on Electronics Computer and Computation, ICECCO 2015, 2016.

4. A. Lundborg, "Text classification of short messages."

5. K. T. Durant and M. D. Smith, "Mining Sentiment Classification from Political Web Logs," 2006.

6. T. Eriksson Author, J. Boye, and J. Lagergren, "Automatic web page categorization using text classication methods Supervisor," 2013.

7. Q. Zhao, X. Cai, C. Chen, L. Lv, and M. Chen, "Commented content classification with deep neural network based on attention mechanism," in Proceedings of 2017 IEEE 2nd AdvancedInformation Technology, Electronic and Automation Control Conference, IAEAC 2017, 2017..

8. S. Lakhotia and X. Bresson, "An experimental comparison of text classification techniques," in Proceedings - 2018 International Conference on Cyberworlds, CW 2018, 2018.

9. A. K. Ameen and B. Kaya, "Spam detection in online social networks by deep learning," in 2018 International Conference on Artificial Intelligence and Data Processing, IDAP 2018, 2019.

10. A. K. Uysal and Y. L. Murphey, "Sentiment Classification: Feature Selection Based Approaches Versus Deep Learning," in IEEE CIT 2017 - 17th IEEE International Conference on Computer and Information Technology, 2017.

11. Z. Wang and B. Song, "Research on hot news classification algorithm based on deep learning," in Proceedings of 2019 IEEE 3rd Information Technology, Networking, Electronic and Automation Control Conference, ITNEC 2019, 2019.

12. A. Kaushik and G. Kaur, "Youtube cookery channels viewers comments in Hinglish," May 2019.

13. AbhishekKaushik, AnchalKaushik, NaithaniSudhanshu "A Study on Sentiment Analysis: Methods and Tools," International Journal of Science and Research (IJSR), vol. 4, no. 12, pp. 287-292, Dec. 2015. 